\title{
Transparent block copolymer thin films for protection of optical elements via chemical vapor deposition
}

\author{
Merve Özpirin, Özgenç Ebil* \\ Department of Chemical Engineering, Izmir Institute of Technology, Urla, Izmir 35430, Turkey
}

\section{A R T I C L E I N F O}

\section{Keywords:}

Polymer

Co-polymers

Thin-film

Initiated chemical vapor deposition

Protective coating

\begin{abstract}
A B S T R A C T
In this study, glycidyl methacrylate and $1 \mathrm{H}, 1 \mathrm{H}, 2 \mathrm{H}, 2 \mathrm{H}$-perfluorodecyl acrylate copolymer p(GMA-co-PFDA) thin-films fabricated via Initiated Chemical Vapor Deposition (iCVD) were investigated as protective coatings on optical BK7 glass substrates and commercial optical filters. Durability tests based on military standards MIL-F48616 and MIL-C-48497A were performed to evaluate performance of coatings for the protection of surfaces of optical elements. Cross-linked p(GMA-co-PFDA) copolymer coatings successfully passed all durability tests showing excellent mechanical properties and protection against humidity, salt water, swelling in water, and resistance to organic solvents while providing excellent adhesion to substrate. iCVD process enabled fine tuning of film morphology, mechanical properties and hydrophobicity by controlling the process parameters. Fabricated films were hydrophobic and highly transparent ( $>98 \%$ ) in the wavelength range from $300 \mathrm{~nm}$ to $1000 \mathrm{~nm}$. Optical transmittance measurements before and after coating process proved that while providing chemical and physical protection, p(GMA-co-PFDA) copolymer thin-films do not cause any detectable change in optical performance of commercial narrow band and wide band filters.
\end{abstract}

\section{Introduction}

Optical and Electro-Optical (EO) systems have a wide range of military and civilian applications. Also, in recent years, the use of EO imaging systems in consumer level applications have substantially increased. During operation, optical elements such as prisms, lenses, filters and mirrors are exposed to quite harsh and unstable operating conditions like sudden changes in temperature and humidity, dust, physical shock, vibration, liquids and vapors, and radiation etc. Therefore, optical elements are prone to physical damage and corrosion leading to reduced optical performance. In most applications, thin coatings are applied to protect the surface from physical and chemical factors. There are two main requirements for protective coatings for optical elements; coating material(s) must not interfere with optical performance of the system, i.e., it must be transparent in the wavelength ranges of interest, and the coating should provide chemical and/ or physical protection to the surface of optical element. In addition, hydrophobicity of the surface is often a desired property for a protective coating [1, 2]. Driven by industrial needs, there have been significant advancements in thin-film coating technologies for improving coating quality, adding new functionalities and reducing fabrication cost [3]. Today, solution based and solventless vapor phase deposition methods are most commonly used techniques for protective coating applications.
Methods like spin-coating or dip-coating, spraying, brushing, electrophoresis, inkjet printing or roll coating are routinely used for sol-gel based inorganic or hybrid coatings. However, traditional sol-gel methods still suffer from weak bonding, low wear-resistance, high permeability, and porosity control issues [4]. Recently, inorganic-organic hybrid coatings have appeared as promising candidates for protective coatings due to their high abrasion resistance, adhesion and corrosion resistance in alkaline medium [5]. These composite coatings are transparent due to small particle sizes of the fillers and can be prepared by sol-gel methods followed by curing via thermal annealing or ultraviolet-radiation [6]. Unfortunately, most methods involving solvent use for the fabrication of protective coatings are not feasible for non-flat substrates and for large-area applications [7]. Variations in coating thickness and morphology, surface roughness, primary particles size and surface wetting issues are still problematic for sol-gel based methods for inorganic and hybrid coatings [8]. Solventless processes such as Physical Vapor Deposition (PVD) and Chemical Vapor Deposition (CVD) do not suffer from surface wetting issues and reproducibly synthesize high quality conformal thin film coatings over complex substrate geometries [9]. Precise thickness control and excellent coating uniformity are main advantages of PVD and CVD fabricated coatings. Titanium nitride, zirconium nitride, silicon nitride, sapphire, calcite, barium fluoride, calcium fluoride, quartz, lithium

\footnotetext{
* Corresponding authors.

E-mail addresses: merveozpirin@iyte.edu.tr (M. Özpirin), ozgencebil@iyte.edu.tr (Ö. Ebil).
} 
fluoride, diamond like carbon coatings and other inorganic oxides are widely used as protective coating materials [9, 10]. However, PVD and CVD methods are usually associated with elevated temperatures and highly energetic deposition environments hindering the applicability due to substrate material limitations. Moderate to high vacuum requirement for the deposition chamber and relatively high cost of precursor materials also lead to higher manufacturing costs compared to non-vacuum methods.

Nevertheless, most optical elements coated with inorganic and hybrid materials can still sustain damage due to physical and chemical factors mentioned above, and their repair usually is expensive and time consuming, if not completely impossible. Instead of using inorganic thin-film coatings for protection of optical elements, tailored polymer thin-films can provide similar protection performance with reduced materials and fabrication cost. Polymer thin-film coatings can also be gently removed from the surface by using suitable solvents, and a fresh coating can be applied. Optical polymers have a high transmittance in visible and near infrared regions, but have strong absorption in the ultraviolet and throughout the infrared [11]. Polymer thin films are generally produced via spin coating or dip coating methods using a polymer solution. However, solvents may significantly limit the substrate type and geometry for the application and may also have a detrimental effect on the film quality. For instance, solvents may dissolve the substrate material or be absorbed by it so they cannot be removed completely from the coatings [12]. Wetting issue leading to a poor film quality is also a commonly encountered problem with spin and dip coating processes. Non-flat and porous substrates also possess a problem for polymer thin-film fabrication using solvents, since it is extremely difficult to achieve continuous conformal coatings with high thickness uniformity over the substrate surface. Therefore, solventless CVD processes become attractive for polymer coatings on non-flat surfaces when thickness uniformity is essential. Coatings of these surfaces are easily performed with processes taken place in vapor phase, and conformal polymer coatings from nanometer to micrometer thicknesses with good uniformity can be achieved [13]. Initiated-Chemical Vapor Deposition (iCVD) represents a suitable alternative for fabrication of polymer thin-films at very low temperatures [13]. iCVD utilizes thermal energy instead of high electrical energy used in Plasma Enhanced CVD technique [14] for initiation of the polymerization reaction, thus it is similar to Hot Wire CVD [15]. iCVD relies on thermal decomposition of an initiator molecule to generate free radicals whereas Hot Wire CVD requires much higher filament temperatures for monomer fragmentation for generation of the radicals for polymerization. Therefore, iCVD is a very gentle process with a filament temperature range between 200 and $400{ }^{\circ} \mathrm{C}$ [16], compared to filaments temperatures $>400^{\circ} \mathrm{C}$ for Hot Wire CVD [17]. In addition to low thermal budget, higher deposition rates can be achieved in iCVD when compared with other CVD methods [1, 18-20].

Here we report optically transparent and hydrophobic thin polymeric films via iCVD for the protection of surfaces of optical elements. Characterization of fabricated coatings, performance tests that are based on Military (MIL) standards MIL-F-48616 and MIL-C-48497A, and application of coatings to commercial narrow band and broad band optical filters are also presented. iCVD fabricated polymer coatings represent a low-cost alternative to inorganic protective coatings fabricated via PVD and CVD methods. Compared to sol-gel based inorganic and hybrid coatings, iCVD fabricated protective coatings offer excellent thickness control over complex substrate geometries with comparable protection performance. In addition, iCVD process can easily be scaled up to accommodate large substrates such as solar panels which is difficult to achieve with current production methods.

\section{Experimental details}

\subsection{Polymer deposition}

Homopolymers of glycidyl methacrylate (pGMA) and $1 \mathrm{H}, 1 \mathrm{H}, 2 \mathrm{H}$, 2H-perfluorodecyl acrylate (pPFDA), and copolymer p(GMA-co-PFDA) thin films were fabricated on a variety of optical materials and crystalline silicon (c-Si). pGMA and pPFDA polymers were selected due to good mechanical properties and high hydrophobicity, respectively [1, 21-23]. All film depositions were performed via iCVD technique. GMA (97\%) and PFDA (97\%) monomers were purchased from Sigma-Aldrich and used without purification. Tert-butyl peroxide (TBPO) (98\%) was also purchased from Sigma-Aldrich and used as initiator.

A custom built iCVD system has been used for deposition of polymer thin films. The vacuum chamber had a square bottom $32 \mathrm{~cm}$ in length and $4 \mathrm{~cm}$ height. The bottom surface had a backside cooled stage $24 \mathrm{~cm}$ in diameter. Temperature of the bottom surface was controlled between -10 to $40{ }^{\circ} \mathrm{C}$ with $0.1^{\circ} \mathrm{C}$ precision by an external circulator (WiseCircu WCR-P8). The reaction chamber was covered with a $2.5 \mathrm{~cm}$ thick quartz plate $25 \mathrm{~cm}$ in diameter. A Nichrome filament $(80 \% \mathrm{Ni} / 20 \% \mathrm{Cr}$ ) array suspended over $2.5 \mathrm{~cm}$ above the cooled stage was used to supply thermal energy for decomposition of the initiator. Filament temperature was measured by a thermocouple (Type K, Omega Engineering) attached to one of the filaments and varied between $230^{\circ} \mathrm{C}$ and $330^{\circ} \mathrm{C}$. Vacuum was provided by a pump (EVP SV-010) connected to a cold trap (Caliskan-Lab 312 CLCT-20R) operated at $-20^{\circ} \mathrm{C}$. Reactor pressure, which was kept between 13.3 and 133 Pa during depositions was controlled by a downstream throttle valve (MKS Type 253B) together with a Baratron capacitance manometer (MKS Type 627D) connected to a pressure controller (MKS Type 651C). Precursor gases were mixed in a separate chamber before entering the reactor. All gas lines and mixing chamber were kept at $80^{\circ} \mathrm{C}$ via heating tapes controlled by electronic controllers to prevent condensation of precursors. Reactor wall temperature was also kept above $40^{\circ} \mathrm{C}$ to promote adsorption of species only on cooled substrate surface. Polymer thin-films were deposited on BK7 glass and other optical substrates with areas up to $80 \mathrm{~cm}^{2}$ at a fixed substrate temperature of $35^{\circ} \mathrm{C}$. A c-Si reference substrate was also used for precise thickness measurements for each deposition. Although iCVD process can reach deposition rates of a few hundred $\mathrm{nm} / \mathrm{min}$, process parameters were intentionally selected to lead much lower deposition rates $(5-10 \mathrm{~nm} / \mathrm{min})$ to improve thickness uniformity. Copolymer $\mathrm{p}$ (GMA-co-PFDA) deposition were performed at a reactor pressure of 26.6 Pa with a PFDA and TBPO flow rates of 0.42 and $1.2 \mathrm{sccm}$, respectively. GMA flow rates were 0.42 and $0.27 \mathrm{sccm}$ for samples $p$ (GMA-co-PFDA)-1 and p(GMA-co-PFDA)-2, respectively. In addition, pGMA and pPFDA homopolymers were also fabricated. The filament temperature was fixed at $300^{\circ} \mathrm{C}$. The average film thickness varied from 20 to $1000 \mathrm{~nm}$. These deposition conditions lead to thickness non-uniformity $<10 \%$ over $80 \mathrm{~cm}^{2}$ substrate surface area.

\subsection{Characterization and testing}

Mprobe-Vis20 reflectometer system with a spectral range of 400-1100 nm was used for film thickness measurements. Before each measurement, the system was calibrated with a reference sample, and all measurements were performed in dark. Fourier Transform Infrared Spectroscopy (FTIR) analysis was performed using a PerkinElmer Inc.Spectrum BX FTIR Spectrometer. Surface roughness and morphology of thin films were evaluated by Nanosurf-Flex Axiom Atomic-Force Microscopy (AFM) system. In addition, Optical Microscopy (OM) study was performed using a BEL MPL-2 polarization microscope for detection of possible surface defects such as pin holes and scratches. A Theta Optical Tensiometer system was used for determination of air/water static contact angles to evaluate hydrophobicity of polymer coatings. Optical transmittance of commercial narrow and wide band filters was measured using an Ocean Optics HR4000CG-UV-NIR Spectrometer. 
MIL-F-48616 and MIL-C-48497A standards which are commonly used for testing optical coatings in defense and aerospace applications were followed for the durability tests of polymer coatings. These standards cover general performance and durability tests of thin film coatings including methods for surface quality (scratches and holes) and surface resistance (adhesion, moisture, solubility in different chemicals, salt resistance and water solubility) evaluation. Humidity tests were performed to determine the resistance of coatings to moisture in operating environment. For humidity tests, substrates coated with both homopolymer and copolymers were placed into a test chamber with a volume of $0.25 \mathrm{~m}^{3}$. The chamber temperature was controlled by external infrared heaters and set to $35^{\circ} \mathrm{C}$ with $80-95 \%$ relative humidity which was controlled by an ultrasonic humidity source inside the chamber. Coatings were kept in the chamber for $24 \mathrm{~h}$. Film thicknesses were measured using a reflectometer before and after moisture exposure. To examine the resistance of coatings to swelling in water, coated substrates were immersed into deionized water at room temperature for $48 \mathrm{~h}$. For salt water solubility test, a $\mathrm{NaCl}$ solution was prepared by using $80 \mathrm{ml}$ water and $3.58 \mathrm{~g} \mathrm{NaCl}$. Then, samples were immersed into the solution at room temperature for $24 \mathrm{~h}$. For adhesion test, a $1.27 \mathrm{~cm}$ wide cellophane tape was pressed on film surface and then removed from the surface at an angle that is normal to the coated surface. Visual inspections and thickness measurements were performed by using optical microscopy and reflectometer, respectively. Film thicknesses were measured using a reflectometer before and after each test. It should be mentioned that MIL-F-48616 and MIL-C-48497A standards were originally designed for inorganic thin film coatings. Still, solubility tests were performed for homopolymer and copolymer film coatings. Polymer film coatings were sequentially immersed into trichloroethylene, acetone and ethyl alcohol for $20 \mathrm{~min}$. To remove excess solvent from the surface, samples were exposed to air for $5 \mathrm{~min}$ without any drying process, and then were immersed in next solution. Film thicknesses before and after the test were measured, and OM analysis was performed after the test for each sample.

\section{Results and discussions}

To determine the optimum thickness of a protective coating for a particular substrate (optical material), optical transmittance of the substrate in the wavelength range of interest must be known. Since the focus of this study was on optical elements used in visible to near infra red region, optical transmittance of pGMA and pPFDA thin-films with various thicknesses on BK7 glass were calculated between 300 and $1000 \mathrm{~nm}$ using TF Companion software. As seen in Fig. 1, BK7 glass has a very high optical transmittance between 300 and $1000 \mathrm{~nm}$ making it a very attractive material for optical applications in visible spectrum. Simulated optical transmittance behaviors of pGMA and pPFDA thin films with thicknesses from $50 \mathrm{~nm}$ to $1000 \mathrm{~nm}$ on BK7 glass substrates are also shown in Fig. 1. Optical transmittance is slightly higher for pPFDA coatings due to lower refractive index $(n=1.36)$ compared to pGMA coatings which has a refractive index varying between 1.4 and 1.5. It was determined that film thickness lower than $1000 \mathrm{~nm}$ does not significantly affect optical transmittance of BK7 glass substrate in visible region. Although fabrication of polymer film as thin as $10 \mathrm{~nm}$ with excellent thickness uniformity is possible via iCVD process, for characterization and durability testing $20 \mathrm{~nm}$ to $500 \mathrm{~nm}$ thick polymer films were fabricated.

FTIR analysis which is routinely used for evaluating the quality of polymer thin-films was performed for GMA, PFDA monomers and polymer films fabricated via iCVD. In FTIR spectra of GMA and PGMA shown in Fig. 2 (top) prominent characteristic peaks at about 3000 and $2932 \mathrm{~cm}^{-1}$ are clearly seen. These peaks belong to $\mathrm{C}-\mathrm{H}$ symmetry and asymmetry stretching caused by $\mathrm{CH}_{3}$ and $\mathrm{CH}_{2}$ groups of GMA and pGMA [1]. Additionally, the distinct peak observed at $1730 \mathrm{~cm}^{-1}$ is assigned to carbonyl group $(\mathrm{C}=\mathrm{O})$ stretching vibration $[22,24]$. The most significant peak at $1640 \mathrm{~cm}^{-1}$ assigned to $\mathrm{C}=\mathrm{C}$ is seen clearly in
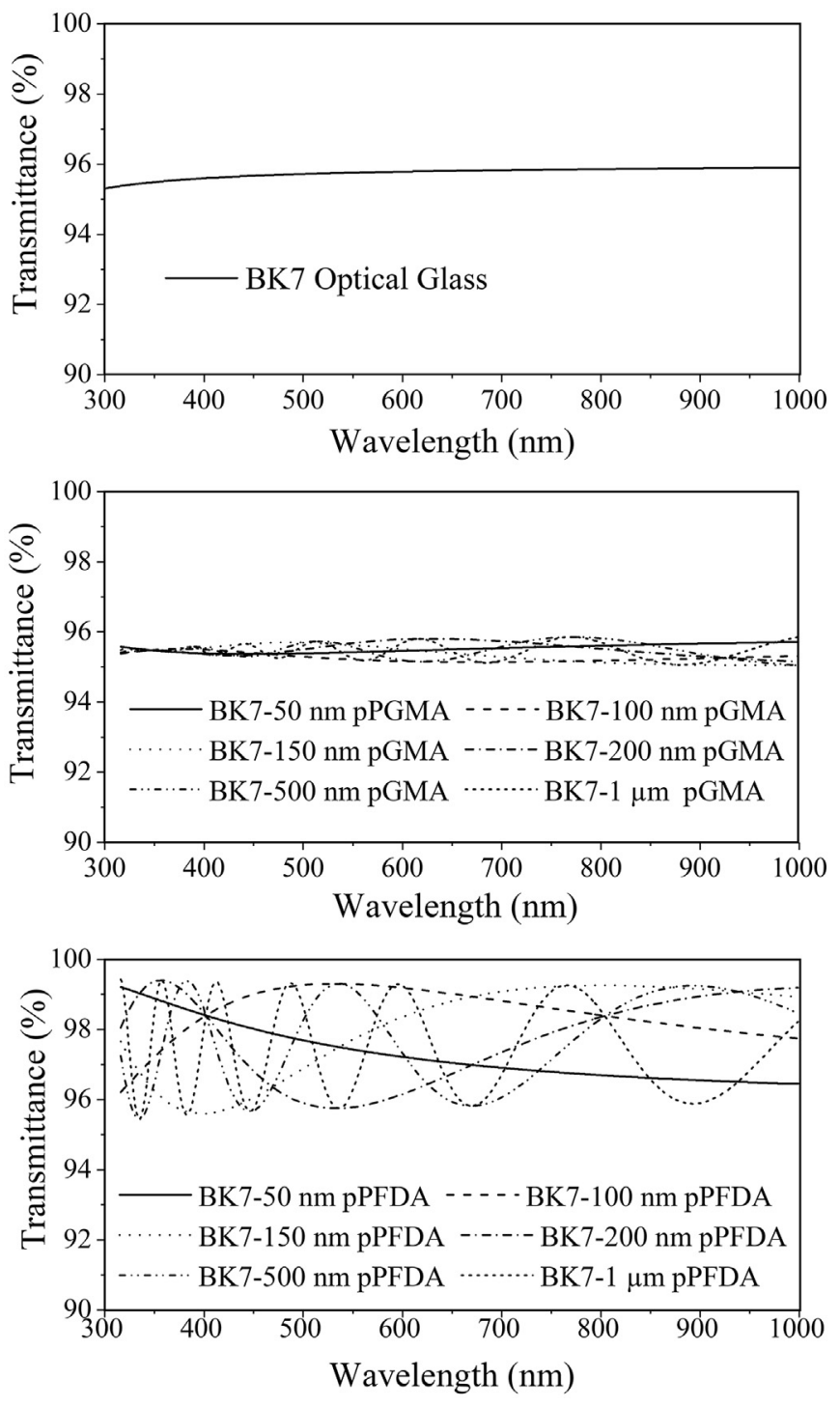

Fig. 1. Simulated optical transmittance of BK7 glass (top), pGMA (middle) and pPFDA (bottom) homopolymers with thickness from $50 \mathrm{~nm}$ to $1000 \mathrm{~nm}$.

GMA spectrum while it is not observed in that of pGMA indicating that no monomer unit is present in the film after polymerization [22]. The characteristic peaks of GMA at 906,846 and $760 \mathrm{~cm}^{-1}$ belong to epoxy group stretching, are clearly seen in both monomer and polymer spectra since functional groups are not affected during polymerization in iCVD process [22, 24-28]. FTIR spectra of PFDA and PPFDA are shown in Fig. 2 (middle). Two specific peaks are present in monomer spectrum around $3020 \mathrm{~cm}^{-1}$ and $2980 \mathrm{~cm}^{-1}$ due to $\mathrm{C}=\mathrm{C}$ double bond. These peaks are not seen in polymer spectrum because unsaturated bonds in monomer are transformed into saturated form during polymerization [29]. The peaks observed at 2970, 2914 and $2864 \mathrm{~cm}^{-1}$ are assigned to $\mathrm{C}-\mathrm{H}$ stretching and $\mathrm{CH}_{2}$ vibrations. The most specific peak seen in both spectra at $1740 \mathrm{~cm}^{-1}$ is associated with carbonyl group $(\mathrm{C}=\mathrm{O})$ stretching $[29,30]$. Furthermore, the characteristic peaks observed in monomer spectrum at 1637, 1620,1413, 1401, 995 and $968 \mathrm{~cm}^{-1}$ are assigned to carbon double bond and are not seen in the polymer spectrum due to complete polymerization of the monomer [29]. Characteristic peaks at 1246,1206 and $1150 \mathrm{~cm}^{-1}$ are clearly observed in both monomer and polymer spectra due to asymmetric, symmetric stretching of $-\mathrm{CF}_{2}-$ and $-\mathrm{CF}_{2}-\mathrm{CF}_{3}$ groups, respectively [29, 31]. FTIR spectra of copolymers having different compositions are given in Fig. 2 (bottom). Due to higher GMA in its composition, FTIR spectrum of 

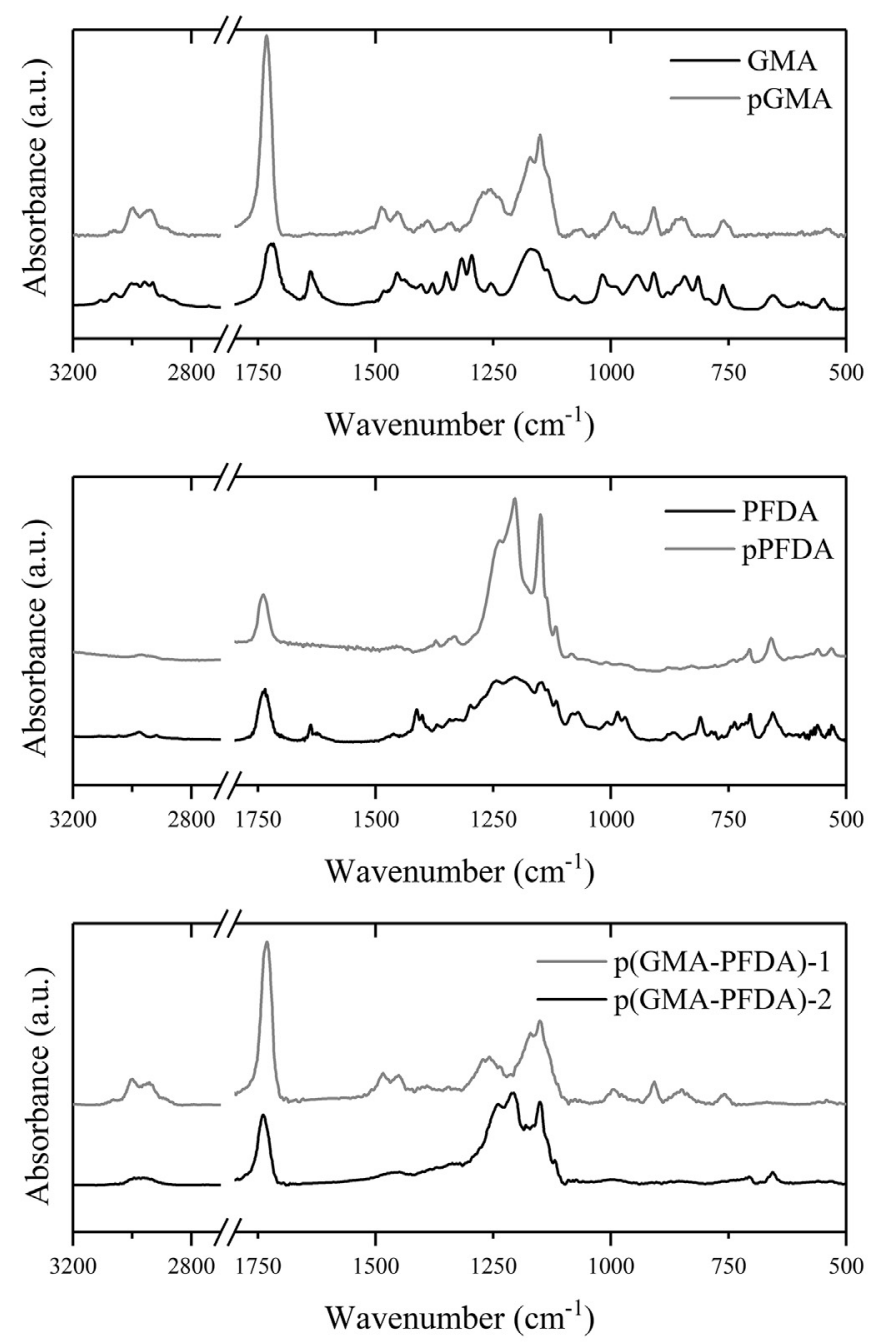

Fig. 2. FTIR spectra of GMA monomer and pGMA film (top), PFDA monomer and pPFDA film (middle), and copolymer p(GMA-co-PFDA) films (bottom) fabricated via iCVD. All spectra are thickness normalized.

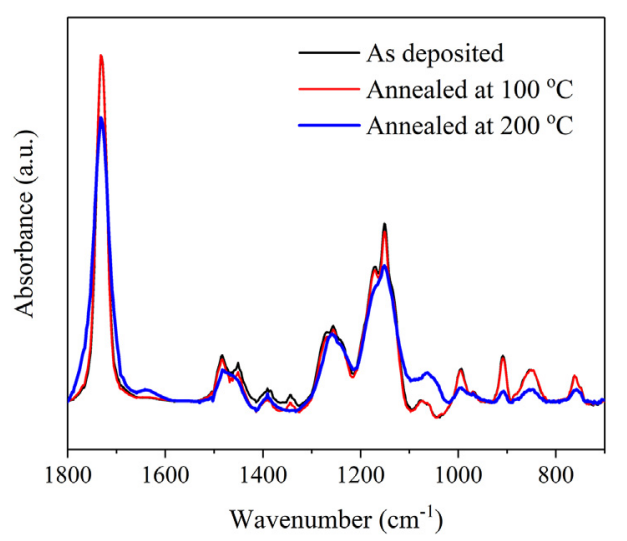

Fig. 3. FTIR spectra (normalized) of pGMA films on c-Si substrate before and after annealing at $100^{\circ} \mathrm{C}$ and $200^{\circ} \mathrm{C}$.

copolymer p(GMA-co-PFDA)-1 film contains epoxy peaks at 908, 847 and $760 \mathrm{~cm}^{-1}$ similar to pGMA. Besides, the characteristic symmetry and asymmetry $\mathrm{C}-\mathrm{H}$ stretching peaks at about $3000 \mathrm{~cm}^{-1}$ are clearly observed. On the other hand, in spectrum of copolymer p(GMA-coPFDA)-2, intensities of epoxy peaks are very low. Characteristic $-\mathrm{CF}_{2}$ and $-\mathrm{CF}_{2}-\mathrm{CF}_{3}$ stretching peaks of pure pPFDA at around 1250 and
$1150 \mathrm{~cm}^{-1}$ are clearly observed in also copolymer p(GMA-co-PFDA)-2 in addition to pPFDA. The ratios of flow rates of GMA and PFDA monomers during polymerization process correlate with the composition of final copolymer films. It has been shown that iCVD pGMA films can crosslink via reaction of the epoxy groups when annealed above $120^{\circ} \mathrm{C}$ in air [32]. This is particularly important since optical elements on EO system routinely experience great variations in temperature and may go through unintentional annealing due to elevated temperatures. Homopolymer pGMA and copolymer films were annealed at different temperatures for $4 \mathrm{~h}$ in air to evaluate the effect of annealing on films thickness. FTIR spectra of pGMA coating before and after annealing at $100{ }^{\circ} \mathrm{C}$ and $200{ }^{\circ} \mathrm{C}$ are shown in Fig. 3. Strong absorption peak assigned to carbonyl group $(\mathrm{C}=\mathrm{O})$ stretching vibration at $1730 \mathrm{~cm}^{-1}$ remains clearly visible [22]. On the other hand, characteristic absorption peaks of epoxy group in pGMA which are at 908,847 and $760 \mathrm{~cm}^{-1}$, almost disappear at $200{ }^{\circ} \mathrm{C}$ indicating epoxy ring-opening reaction for crosslinking [27]. Films annealed at temperatures above $220^{\circ} \mathrm{C}$ showed signs of thermal degradation.

MIL-F-48616 and MIL-C-48497A standards establish certain requirements for single or multilayer coatings on optical elements related to physical appearance, environmental and solubility blemishes, surface defects etc. In some cases, MIL standards do not provide measurable limits but do provide general guidelines for visual inspection. Surface defects on coatings such as scratches and pin holes which may not be visible during visual inspection can be seen under optical microscope, and defect density can be calculated. Surface morphologies of pGMA and PPFDA, and copolymer coatings on optical substrates were examined for possible surface defects by optical microscope using polarization filters. Fig. 4 shows OM images of copolymers deposited on BK7 glass substrates. A uniform surface morphology was observed for polymeric film fabricated via iCVD process. Although not shown in Fig. 4, pPFDA films has some scratches around the edges of the substrates as a result of mechanical handling of the samples. This is due to poor mechanical properties of PFDA films and is not related to iCVD process. Although substrates and vacuum chamber were cleaned before every deposition, some dust particles were also observed on film surface. It is reasonable to assume that during the transport of the samples from reaction chamber to the sample box, dust particles in the laboratory environment stick to freshly coated surface before annealing process. As shown in Fig. 4a and b, uniform coatings were obtained for both p(GMA-co-PFDA) films. The surface of copolymer p(GMA-coPFDA)-2 was observed to be rougher than that of copolymer p(GMA-coPFDA)-1 due to higher PFDA content. In both copolymer films, no visible scratches, pin holes or other defects due to handling were observed. This is a good indication of improved mechanical properties of copolymer films compared to pure fluoropolymer due to incorporation of GMA. MIL-F-48616 standard requires that the total length of all scratches on the coating surface should be $<25 \%$ of the diameter of the coated substrate. In addition, the sum of the products of the widths designated by the scratch letters times the ratio of their length to diameter of the coated surface should not exceed the width specified by the scratch letter where scratch letters A, B, C, D, E, F and G are specified as having a scratch width of $5,10,20,40,60,80$, and $120 \mu \mathrm{m}$, respectively. The total length of the scratches observed at the edges of pPFDA coated substrates were determined to be $<15 \%$ of the diameter of the substrate while for pGMA and p(GMA-co-PFDA) coatings this number was always $<1 \%$. Film morphology and surface uniformity of copolymer coatings on c-Si and BK7 glass substrates were examined with Scanning Electron Microscope (SEM). Fig. 5 shows SEM images of $p$ (GMA-co-PFDA) films with different compositions. SEM analysis revealed that iCVD deposited films showed uniform surface morphologies over entire substrate surface without any defects. As seen in Fig. 5a, copolymer p(GMA-co-PFDA)-1 showed a surface morphology similar to pGMA films with bead-like structures as previously reported in literature [13]. It was claimed that this morphology is a result of high amount of radical generation due to high filament temperature 


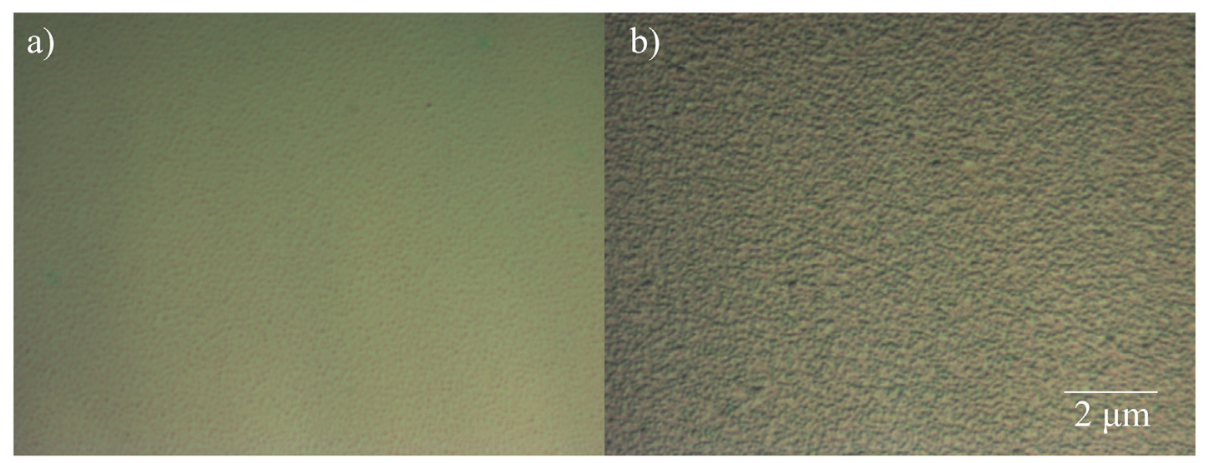

Fig. 4. Optical microscopy images of a) p(GMA-co-PFDA)-1 and, b) p(GMA-co-PFDA)-2 copolymer films at 80X magnification under top illumination.

$\left(300-330^{\circ} \mathrm{C}\right)[25]$. On the other hand, in Fig. 5b, copolymer p(GMA-coPFDA)-2 film surface appears prominently rough and compact. The difference between copolymer film morphologies is a result of differences between compositions of copolymer films resulting from varying monomer flow rate ratios used in process. No major defects that can interfere with the optical transmittance were observed.

Surface roughness is one of the most important parameters for optical materials affecting optical performance. Usually, very smooth surfaces for mirrors, lenses and prisms are only achieved after careful grinding and polishing processes. However, these processes are mostly applicable to inorganic materials; therefore, surface roughness of polymeric thin film coatings must be controlled during polymerization/ deposition process. Fortunately, iCVD process enables smooth conformal coatings on any flat substrate [1]. AFM analysis performed on iCVD fabricated thin films confirmed that iCVD process indeed led to conformal coatings with relatively smooth surfaces on variety of substrates. Fig. 6 shows AFM surface maps of $10 \mu \mathrm{m}$ by $10 \mu \mathrm{m}$ area of approximately $500 \mathrm{~nm}$ thick p(GMA-co-PFDA) thin-films on c-Si substrates. Root mean square (RMS) roughness, Rq, of c-Si substrate was $1.09 \pm 0.2 \mathrm{~nm}$. Rq value of copolymers p(GMA-co-PFDA)-1 (Fig. 6a) and p(GMA-co-PFDA)-2 (Fig. $6 \mathrm{~b}$ ) were found as $16.2 \mathrm{~nm} \pm 3.1 \mathrm{~nm}$ and $30.0 \pm 4.3 \mathrm{~nm}$, respectively. Surface morphology correlates well with SEM observations as copolymer p(GMA-co-PFDA)-1 has higher GMA in its composition leading to a smoother surface. In addition, Rq values for pGMA and pPFDA homopolymer films fabricated via iCVD on c-Si substrates were determined to be $14.94 \pm 2.3 \mathrm{~nm}$ and $22.48 \pm 3.7 \mathrm{~nm}$, respectively. These values also agree well with the previous studies in literature $[22,29]$. It was also observed that surface roughness decreases significantly with decreasing p(GMA-co-PFDA) film thickness. Therefore, to avoid any degradation of optical performance due to scattering, polymer film thickness should be limited to less than a few micrometers.

For protective coatings on optical elements, hydrophobicity is one of the most desired features. As a quantitative measure of hydrophobicity of a surface, static water contact angle (WCA) measurements were performed for copolymer thin films as well as homopolymers of pGMA and pPFDA. Fig. 7 shows droplet images captured during WCA measurements on $\mathrm{c}-\mathrm{Si}$ substrates coated with pGMA, pPFDA and $\mathrm{p}$ (GMA-co-PFDA) films before and after annealing at $200^{\circ} \mathrm{C}$ for $2 \mathrm{~h}$. As expected, pPFDA films were hydrophobic with an average contact angle of $132^{\circ}$ because of fluorine containing groups whereas hydrophilic pGMA films showed a contact angle of $64^{\circ}$. Due to higher fluorine content, copolymer p(GMA-co-PFDA)-2 had a WCA of $126^{\circ}$, greater than that of copolymer p(GMA-co-PFDA)-1 ( $85^{\circ}$ ). Obviously, the surface of p(GMA-co-PFDA)-1 coating is not hydrophobic making it not suitable for optical coating applications. Annealing of coatings resulted in reduced surface roughness and hydrophobicity. As seen in Fig. 7, annealing copolymer p(GMA-co-PFDA)-2 film at $200^{\circ} \mathrm{C}$ for $2 \mathrm{~h}$ reduced WCA from $126^{\circ}$ to $120.4^{\circ}$. While copolymerization decreases water wettability of the films due to lower fluorine content, it helps to improve mechanical properties of the fluoropolymer coatings. The hydrophobicity of the films can be tuned during polymerization in iCVD process by adjusting the flow rates of monomers [23, 33]. Since pPFDA films have lower mechanical strength than pGMA films, optimization of hydrophobicity and chemical/mechanical properties of copolymer films should be based on particular application environment.

To evaluate the performance of fabricated films as protective coatings on optical elements, MIL-F-48616 and MIL-C-48497A standards were used. These specifications establish general performance and durability requirements for inorganic coatings on optical materials, but some of the tests are also applicable to polymeric thin film coatings. Humidity, swelling in water, salt resistance, adhesion and solubility tests were performed as described in standards. However, the test protocol does not specify any measurable criteria but states that after immersion in organic solvents, coated substrates should show no evidence of coating removal or scratches. Film thicknesses before and after the tests were measured using a reflectometer that had a $2 \mathrm{~mm}$ spot size and $2 \mathrm{~nm}$ measurement accuracy. Films were also evaluated visually and under optical microscope for possible defects. The differences in measured thicknesses of the films before and after the tests were within $\mp 2 \mathrm{~nm}$ indicating no swelling or dissolving of the films. These tests show that fabricated coatings are resistant to humidity, and immersion in water and salt water solution. To evaluate the adhesion of the polymeric coatings onto a variety of optical materials, adhesion tests

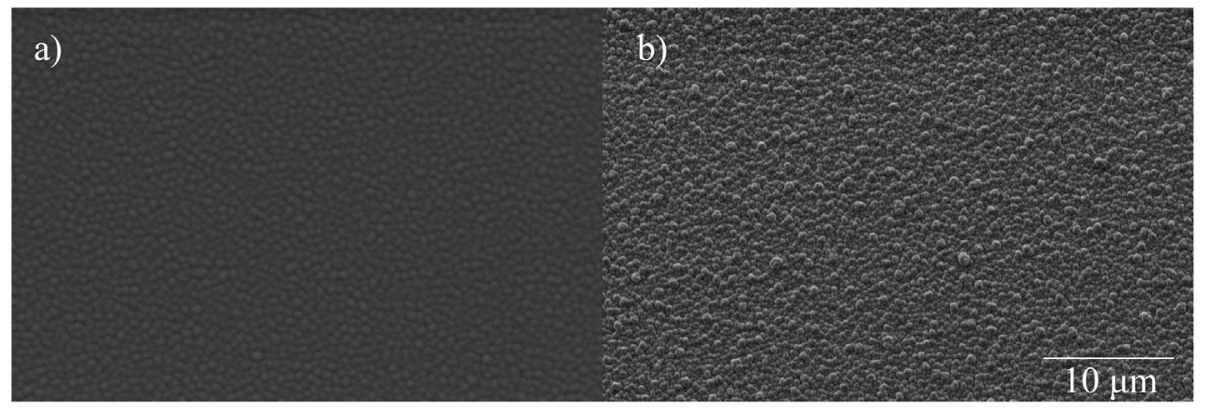

Fig. 5. SEM images of a) p(GMA-co-PFDA)-1, and (d) p(GMA-co-PFDA)-2 copolymer films fabricated on c-Si substrate. 


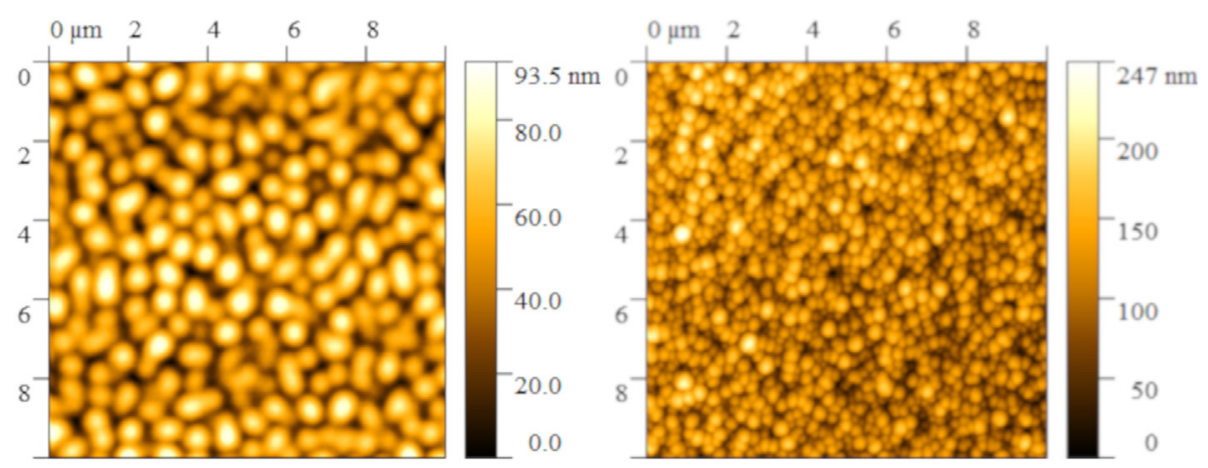

Fig. 6. AFM analysis of approximately $500 \mathrm{~nm}$ thick a) p(GMA-co-PFDA)-1, and b) p(GMA-co-PFDA)-2 copolymer films. Sample area is $10 \mu \mathrm{m}$ by $10 \mu \mathrm{m}$.

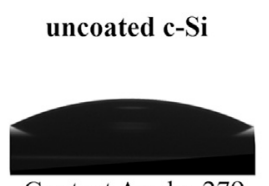

Contact Angle: 270

p(GMA-co-PFDA)-1

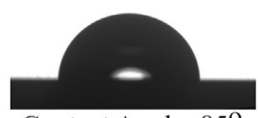

Contact Angle: $85^{\circ}$

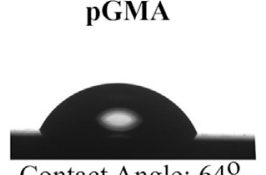

Contact Angle: $64^{\circ}$

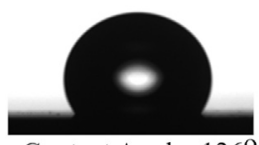

Contact Angle: $126^{\circ}$
p(GMA-co-PFDA)-2

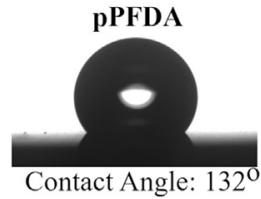

p(GMA-co-PFDA)-2 annealed

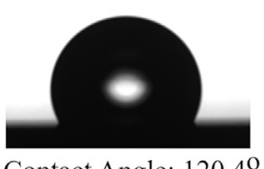

Contact Angle: $120.4^{\mathrm{O}}$
Fig. 7. Water droplet images captured during air/water contact angle measurements of polymer coatings on c-Si substrate.

were also performed. Before and after the tests, visual inspections and thickness measurements were performed using an optical microscope and a reflectometer, respectively. Thicknesses of p(GMA-co-PFDA) copolymer films before and after the adhesion test, were again within $\mp$ $2 \mathrm{~nm}$. No defects were observed with OM analysis on the surface of copolymer p(GMA-co-PFDA)-1 after adhesion test. Copolymer p(GMAco-PFDA)-1 survived repeated adhesions tests without any damage. However, a small piece of copolymer p(GMA-co-PFDA)-2 film was removed by the cellophane tape from the surface during adhesions tests as seen in Fig. 8. This was not surprising as the mechanical properties of this copolymer film was expected to be poorer than copolymer p(GMAco-PFDA)-1 film due to higher amount of fluorine groups. Compared to total area of the film tested for adhesion, the area of piece of film that was removed from the surface is still within acceptable range by MIL-F48616 and MIL-C-48497A standards. The mechanical strength, hydrophobicity and adhesion of the film to the substrate surface can be optimized for a particular application by carefully controlling the

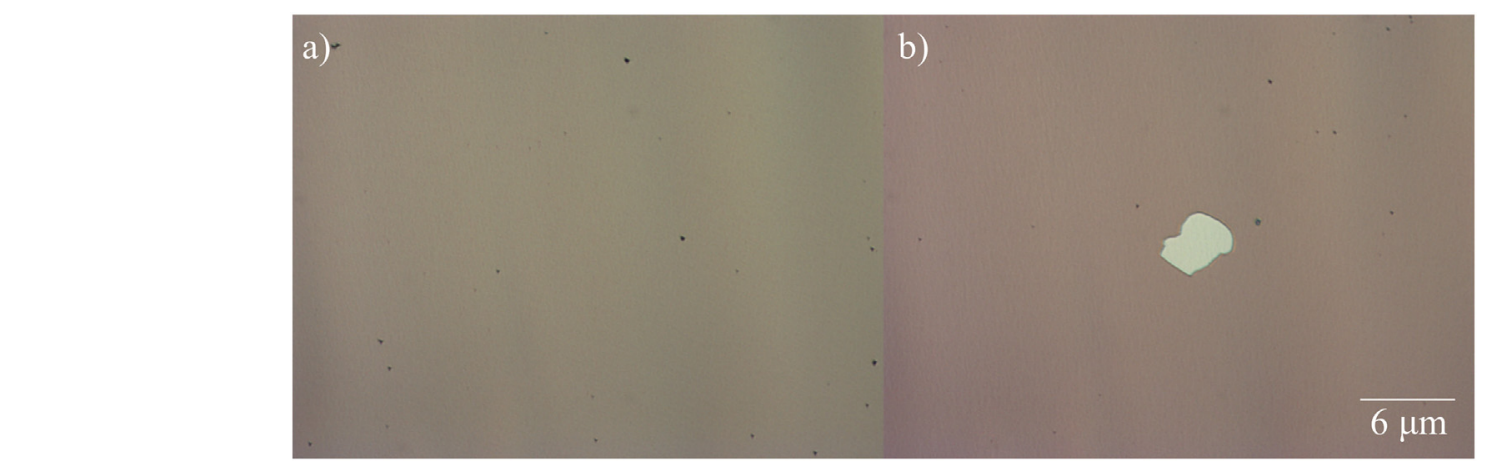

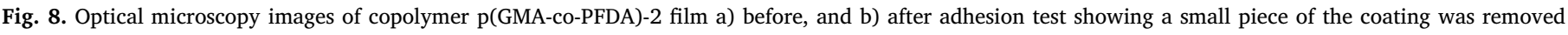
during the test. copolymer film composition. To determine the resistance of polymeric coating against organic solvents, a test protocol involving trichloroethylene, acetone and ethyl alcohol as organic solvents was followed. As expected homopolymer films and copolymer films without annealing (cross-linking) were dissolved completely. Copolymer films cross-linked by annealing at $200{ }^{\circ} \mathrm{C}$ for $2 \mathrm{~h}$ successfully passed all solubility tests without any observable change in film morphology, thickness and contact angle.

Copolymer films were also applied to outer surfaces of several commercially available optical filters. An Oxygen III narrow band filter that isolates two doubly ionized oxygen lines at 496 and $501 \mathrm{~nm}$, and a light pollution filter that blocks emission lines of artificial light sources like streetlights as well as the airglow but passes light from 450 to $520 \mathrm{~nm}$ and from 640 to $690 \mathrm{~nm}$ were coated with copolymer p(GMAco-PFDA)-2 films. An average film thickness of $20 \mathrm{~nm}$ to $30 \mathrm{~nm}$ was targeted to minimize the surface roughness of the coating. Normalized optical transmittance of filters before and after coating process are shown in Fig. 9. Two emission peaks at 496 and $501 \mathrm{~nm}$ in Oxygen III filter spectrum did not vary in absolute transmittance $(93.6 \%$ and $94.2 \%$, respectively) after coating the filter but the background noise increased approximately $2 \%$. Light pollution filter has two pass regions expanding from 450 to $520 \mathrm{~nm}$ and from 640 to $690 \mathrm{~nm}$ with absolute transmittance of approximately $88 \%$ and $95 \%$, respectively. Noise in data was increased slightly, but protective coating did not change transmittance behavior of the filter. We did not observe any change in the maximum transmittance of the filters since multi-layered narrow band and broad band optical filters in visible region have $\mathrm{MgF}_{2}$ antireflective layers with an index of refraction of 1.38 at $550 \mathrm{~nm}$ matching well with the refractive index of copolymer coating.

\section{Conclusions}

Copolymer $\mathrm{p}$ (GMA-co-PFDA) thin-film coatings were fabricated via iCVD process on optical substrates for protection of the surface from 

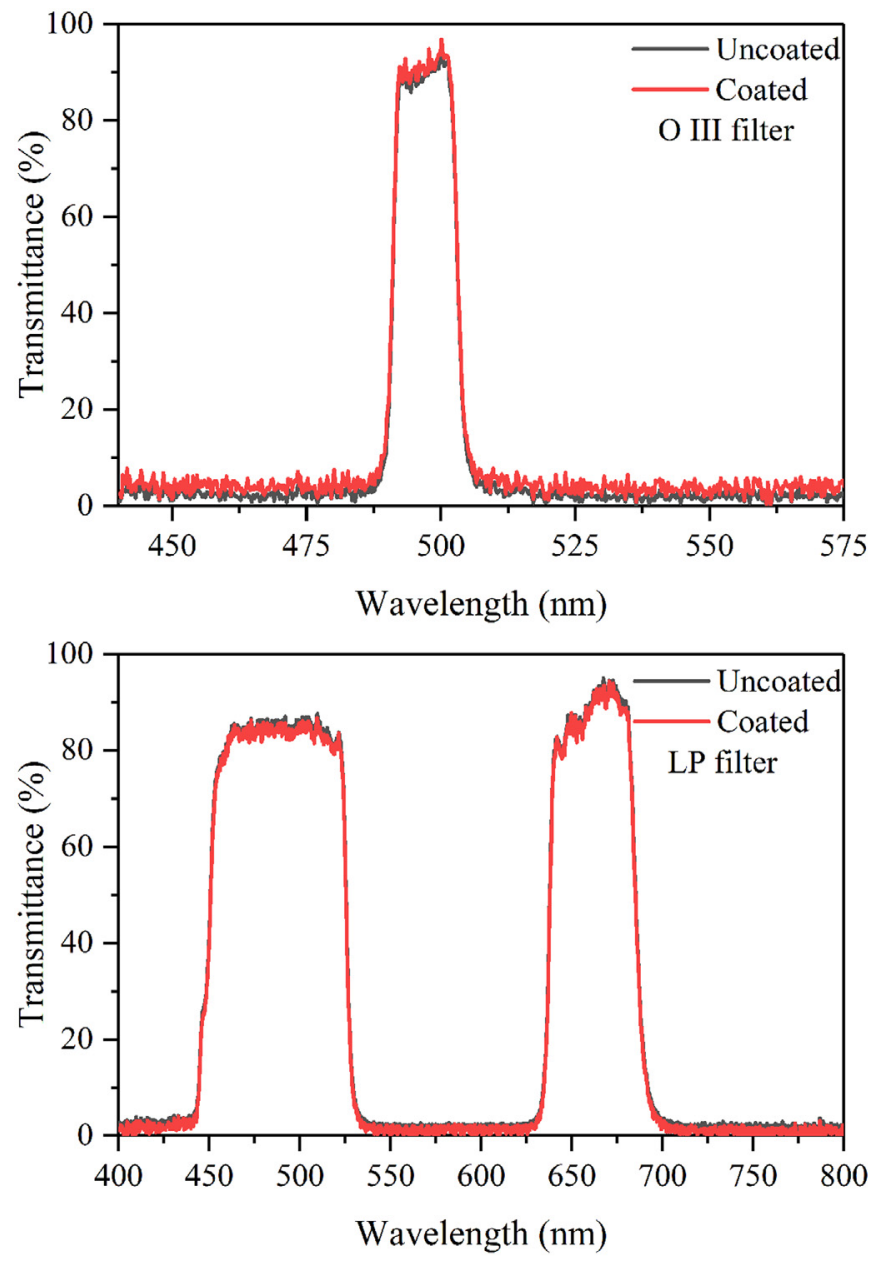

Fig. 9. Optical transmittance (normalized) of oxygen III narrow band filter (top) and light pollution filter (bottom) before and after coating with p(GMAco-PFDA) thin-films.

harsh environmental conditions. Feasibility of fabricated copolymer thin films as protective coatings in optical systems was confirmed by durability tests based on MIL-F-48616 and MIL-C-48497A standards. While pGMA homopolymer films have excellent mechanical and optical properties, hydrophobicity of the surface is not sufficient to be used as protective optical coating. On the other hand, pPFDA homopolymer films show excellent surface hydrophobicity but are prone to scratches and other surface damage during mechanical handling due to weak mechanical properties. Copolymer p(GMA-co-PFDA) coatings showed excellent mechanical properties and protection against water and organic solvents combining enhanced mechanical properties of pGMA and hydrophobicity of pPFDA. They also showed high resistance to humidity, salt solubility, swelling and solubility in water. Optical and mechanical properties of copolymers can be finely tuned by controlling the flow rates of monomers during iCVD deposition process, followed by a low temperature annealing process for cross-linking to further improve chemical resistance and adhesion of the coatings without sacrificing optical performance. Application of p(GMA-co-PFDA) thin-film coatings via iCVD onto commercially available narrow band and broad band optical filters did not change transmittance of light or cause any detectable optical performance decrease while providing chemical and physical protection from the elements. It can be concluded that iCVD fabricated polymer coatings offer a low-cost alternative to current solgel and CVD based inorganic and hybrid protective coatings for optical elements. All-polymer protective coatings via iCVD combine scalability of CVD processes with excellent control of film morphology and thickness over complex substrate geometries.

\section{Acknowledgements}

This work was supported by the Scientific and Technological Research Council of Turkey (TÜBITTAK) [Grant Number 114M233] and Marie Curie Career Integration Grants (CIG) [Grant Number PCIG11GA-2012-321,692]. We also thank Dr. Sinan Balc1 and Dr. Emre Sarı for their help with optical characterization.

\section{Notes}

The authors declare no competing financial interest.

\section{References}

[1] K.K. Gleason, CVD Polymers: Fabrication of Organic Surfaces and Devices, Wiley, 2015.

[2] K.K.S. Lau, K.K. Gleason, Particle functionalization and encapsulation by initiated chemical vapor deposition (iCVD), Surf. and Coat.Technol. 201 (2007) 9189-9194.

[3] A. Piegari, F. Flory, Optical Thin Films and Coatings, Woodhead Publishing, 2013.

[4] M. Pagliaro, R. Ciriminna, G. Palmisano, Silica-based hybrid coatings, J. Mater. Chem. 19 (2009) 3116-3126.

[5] I.J. Zvonkina, M.D. Soucek, Inorganic-organic hybrid coatings: common and new approaches, Current Opinion in Chemical Engineering. 11 (2016) 123-127.

[6] K.H. Haas, S. Amberg-Schwab, K. Rose, Functionalized coating materials based on inorganic-organic polymers, Thin Solid Films 351 (1999) 198-203.

[7] R. Taurino, E. Fabbri, D. Pospieech, A. Synytska, M. Messori, Preparation of scratch resistant superhydrophobic hybrid coatings bysol-gel process, Prog. Org. Coat. 77 (2014) 1635-1641.

[8] D. Ebert, B. Bhushan, Transparent, superhydrophobic, and wear-resistant Coatings on glass and polymer substrates using $\mathrm{SiO}_{2}, \mathrm{ZnO}$, and ITO nanoparticles, Langmuir 28 (2012) 11391-11399.

[9] S.H. Mousavi, M.H. Jilavi, M. Koch, E. Arzt, P.W. Oliveira, Development of a transparent scratch resistant coating through direct oxidation of Al-coated glass, Adv. Eng. Mater. 19 (2017) 1600617.

[10] M. Wakaki, Optical Materials and Applications, Taylor \& Francis, 2012.

[11] F. Träger, Springer Handbook of Lasers and Optics, Springer, 2012.

[12] M.E. Alf, A. Asatekin, M.C. Barr, S.H. Baxamusa, H. Chelawat, G. Ozaydin-Ince, C.D. Petruczok, R. Sreenivasan, W.E. Tenhaeff, N.J. Trujillo, S. Vaddiraju, J. Xu, K.K. Gleason, Chemical vapor deposition of conformal, functional, and responsive polymer films, Adv. Mater. 22 (18) (2010) 1993-2027.

[13] T.C. Parker, D. Baechle, J.D. Demaree, Polymeric barrier coatings via initiated chemical vapor deposition, Surf. and Coat.Technol. 206 (2011) 1680-1683.

[14] H.O. Pierson, Handbook of Chemical Vapor Deposition, Second ed., Principles, Technology and Applications, Elsevier Sci, 1999 pp. 134-143.

[15] K. Chan, L.E. Kostun, W.E. Tenhaeff, K.K. Gleason, Initiated chemical vapor deposition of polyvinylpyrrolidone-based thin films, Polymer 47 (2006) 6941-6947.

[16] K.K.S. Lau, K.K. Gleason, Particle functionalization and encapsulation by initiated chemical vapor deposition (iCVD), Surf. and Coat.Technol. 201 (2007) 9189-9194.

[17] A.M. Coclite, Smart surfaces by initiated chemical vapor deposition, Surf. Innovations. 1 (2013) 6-14.

[18] A.M. Coclite, Y. Shi, K.K. Gleason, Super-Hydrophobic and Oloephobic Crystalline Coatings by Initiated Chemical Vapor Deposition, Phys. Procedia 46 (2013) 56-61.

[19] K.K.S. Lau, K.K. Gleason, Initiated chemical vapor deposition (iCVD) of poly (alkyl acrylates): An experimental study, Macromolecules 39 (2006) 3688-3694.

[20] K.K.S. Lau, K.K. Gleason, Applying HWCVD to particle coatings and modeling the deposition mechanism, Thin Solid Films 516 (2008) 674-677.

[21] T.P. Martin, K.K.S. Lau, K. Chan, Y. Mao, M. Gupta, A.S. O'Shaughnessy, K.K. Gleason, Initiated chemical vapor deposition (iCVD) of polymeric nanocoatings, Surf. and Coat.Technol. 201 (2007) 9400-9405.

[22] R. Bakker, V. Verlaan, C.H.M. van der Werf, J.K. Rath, K.K. Gleason, R.E.I. Schropp, Initiated chemical vapour deposition (iCVD) of thermally stable poly-glycidyl methacrylate, Surf. and Coat.Technol. 201 (2007) 9422-9425.

[23] M. Gupta, V. Kapur, N.M. Pinkerton, K.K. Gleason, Initiated Chemical Vapor Deposition (iCVD) of Conformal Polymeric Nanocoatings for the Surface Modification of High-Aspect-Ratio Pores, Chem. Mater. 20 (2008) 1646-1651.

[24] S. Mohammed Safiullah, K. Abdul Wasi, K. Anver Basha, Preparation of poly (Glycidyl methacrylate)-copper nanocomposite by in-situ suspension polymerization - A novel synthetic method, Mater. Lett. 133 (2014) 60-63.

[25] Y. Mao, K.K. Gleason, Hot Filament Chemical Vapor Deposition of Poly(glycidyl methacrylate) Thin Films Using tert-Butyl Peroxide as an Initiator, Langmuir 20 (2004) 2484-2488

[26] M. Gupta, K.K. Gleason, Large-scale initiated chemical vapor deposition of poly (glycidyl methacrylate) thin films, Thin Solid Films 515 (2006) 1579-1584.

[27] Y. Mao, K.K. Gleason, Vapor-Deposited Fluorinated Glycidyl Copolymer Thin Films with Low Surface Energy and Improved Mechanical Properties, Macromolecules 39 (2006) 3895-3900.

[28] S.W. Park, D. Lee, H.R. Lee, H.-J. Moon, B.R. Lee, W.-K. Ko, S.-J. Song, S.J. Lee, K. Shin, W. Jang, J.-K. Yi, S.G. Im, I.K. Kwon, Generation of functionalized polymer nanolayer on implant surface via initiated chemical vapor deposition (iCVD), J. Colloid Interface Sci. 439 (2015) 34-41.

[29] M. Gupta, K.K. Gleason, Initiated Chemical Vapor Deposition of Poly $(1 \mathrm{H}, 1 \mathrm{H}, 2 \mathrm{H}, 2 \mathrm{H}-$ 
perfluorodecyl Acrylate) Thin Films, Langmuir 22 (2006) 10047-10052.

[30] Y.J. Luis, K.K. Gleason, Enhanced Cross-Linked Density by Annealing on Fluorinated Polymers Synthesized via Initiated Chemical Vapor Deposition To Prevent Surface Reconstruction, Macromolecules 46 (2013) 6548-6554.

[31] Y. Yoo, J.B. You, W. Choi, S.G. Im, A stacked polymer film for robust superhydrophobic fabrics, Polym. Chem. 4 (2013) 1664-1671.
[32] V.J.B. Jeevendrakumar, D.N. Pascual, M. Bergkvist, Wafer Scale Solventless Adhesive Bonding with iCVD Polyglycidylmethacrylate: Effects of Bonding Parameters on Adhesion Energies, Adv. Mater. Interfaces 2 (2015) 1500076.

[33] M. Gupta, K.K. Gleason, Surface modification of high aspect ratio structures with fluoropolymer coatings using chemical vapor deposition, Thin Solid Films 517 (2009) 3547-3550. 